3

4$$
5
$$

\title{
A Powerful Approach for Identification of Differentially Transcribed mRNA Isoforms
}

Department of Molecular Physiology and Biophysics and Dan L. Duncan Cancer Center, Baylor College of Medicine, Houston, Texas, 77030

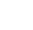
(1) 10 1

2

3

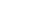

\author{
Yuan-De Tan and Joel. R. Neilson
}


21 Next generation sequencing is being increasingly used for transcriptome-wide

22 analysis of differential gene expression. The primary goal in profiling expression is

23 to identify genes or RNA isoforms differentially expressed between specific

24 conditions. Yet, the next generation sequence-based count data are essentially

25 different from the microarray data that are continuous type, therefore, the

26 statistical methods developed well over the last decades cannot be applicable. For

27 this reason, a variety of new statistical methods based on count data of transcript

28 reads has been correspondingly developed. But currently the transcriptomic count

29 data coming only from a few replicate libraries have high technical noise and small

30 sample size bias, performances of these new methods are not desirable. We here

31 developed a new statistical method specifically applicable to small sample count

32 data called mBeta t-test for identifying differentially expressed gene or isoforms on

33 the basis of the Beta t-test. The results obtained from simulated and real data

34 showed that the mBeta t-test method significantly outperformed the existing

35 statistical methods in all given scenarios. Findings of our method were validated by

36 qRT-PCR experiments. The mBeta t-test method significantly reduced true false

37 discoveries in differentially expressed genes or isoforms so that it had high work

38 efficiencies in all given scenarios. In addition, the mBeta t-test method showed high

39 stability in performance of statistical analysis and in estimation of FDR. These

40 strongly suggests that our mBeta t-test method would offer us a creditable and

41 reliable result of statistical analysis in practice. 
44 Development of high-throughput sequencing technologies in recent years (Cloonan et al.

45 2008a; Cloonan et al. 2008b; Mortazavi et al. 2008)has massively been increasing genomic

46 data and led sequencing cost to rapidly go down so that the sequencing technologies as

47 platforms for studying gene expression or sub-gene expression have become more and

48 more attractive (McCarthy et al. 2012). Current next generation sequencing (NGS)

49 technologies such as RNA-seq (Cloonan et al. 2008a; Cloonan et al. 2008b; Mortazavi et al.

50 2008), Tag-seq (Morrissy et al. 2009), deepSAGE ( $t$ Hoen et al. 2008), SAGE-seq (Wu et al.

51 2010), and PAS-seq (Shepard et al. 2011) can generate short reads of sequence tags, that is,

52 sequences of 35-300 bp that correspond to fragments of the original RNA. In particular, 3P-

53 seq or PAS-seq(Shepard et al. 2011), a deep sequencing-based method for quantitative and

54 global analysis of RNA polyadenylation has been used to study expression behavior of RNA 55 isoforms in a variety of human and mouse cells.

56

57 To evaluate differential expression between conditions or cases, sequences need to be 58 mapped to genome and annotated. After doing so, the sequence data can be transformed to 59 count data at genomic level of interest. Although RNA-Seq can be used to study differential 60 transcription of novel exons, splice-variants and isoforms-specific (Denoeud et al. 2008; Li

61 et al. 2010; Pan et al. 2008; Wang et al. 2009) and allele-specific expression(Degner et al.

62 2009; Montgomery et al. 2010), our focus here is on differential expression of genes or

63 isoforms due to alterative polyadenylation signals and cleavage sites in 3' untranslated 64 regions (3'UTR) . 
66 A RNA sample may be thought of as a RNA population and each RNA sequence as one

67 individual. Sequencing a RNA sequence is a random process of sampling from a RNA

68 population. If each individual RNA has equal chance to be selected for sequencing, then

69 probability of sequencing a RNA sequence is proportional to the length of waiting time

70 (Anders et al. 2010). Thus number of RNA read counts for a given genomic feature should

71 follow Poisson distribution. The Poisson distribution implicates that only one parameter

72 determines count variation of reads. However, since the Poisson model is just with respect

73 to noise but does not deal with biological source of variation, that is, difference in

74 transcription between samples, some counts are over-dispersed between samples under

75 the Poisson Model. Accordingly, variation of read counts consists of two parts:

76 noise(Poisson) and biological variability, that is, $\sigma^{2}=\sigma_{\text {noise }}^{2}+\sigma_{\text {biological }}^{2}=\mu+a \mu^{2}$ where

$77 \sigma_{\text {noise }}^{2}=\mu$ is variance of Poisson distribution and $\sigma_{\text {biological }}^{2}$ is biological variance, which is

78 determined by biological effect $a$. This is just characteristic of negative binomial 79 distribution (Anders et al. 2010; Robinson et al. 2008).

81 To identify differential transcription of RNA tags, many statistical methods have been 82 proposed so far. At early stage, most of methods are based on Poisson distribution 83 (Madden et al. 1997) or normal approximations (Kal et al. 1999; Man et al. 2000; Michiels 84 et al. 1999), permutation (Zhang et al. 1997), beta distribution (Baggerly et al. 2003) or 85 over-dispersed logistic linear distribution (Baggerly et al. 2004). As RNA count data have 86 become more and more prevalent, newer statistical methods such as Exact test(Robinson 
87 et al. 2008), empirical Bayesian method (Hardcastle et al. 2010), DESeq (Anders et al. 2010), generalized linear modeling (McCarthy et al. 2012), and likelihood ratio tests (Wang et al. 2010) have recently been developed.

91 Despite the development of technologies decreasing costs of sequencing, RNA-Seq experiments still remain expensive for many researchers so that RNA-Seq studies have to be limited to only a very small number of replicate libraries for each condition or case. The basic scientific need to assess differential transcription due to biological variation remains undiminished but the problem becomes complicated by the fact that different genes or transcripts may have different degrees of biological variation. There is therefore a need to estimate biological variation as reliably as possible from a few replicate libraries (McCarthy et al. 2012). The classical statistical methods are not applicable for such data. To address this problem, many methods such as empirical Bayesian method (baySeq) (Hardcastle et al. 2010), DESeq (Anders et al. 2010) and Exact test (Robinson et al. 2010b; Robinson et al. 101 2007; Robinson et al. 2008) adopt variation information across transcriptome and the GLM (McCarthy et al. 2012)uses similarity information between genes. However, none of these methods considers fudge effect of small sample size. So-called fudge effect is such an effect on which differences between two means are small but sample variances are also much smaller such that statistics are inflated (Tusher et al. 2001, Tan et al 2007). This is because sample size is so small that there is a big chance to give rise to small difference among replicates in a large-scale data (see Discussion Section for more detail). The fudge 
109 because there are a lot of very small counts. Therefore, to suppress such an effect can 110 greatly improve performance of the statistical methods in identification of differentially 111 expressed genes or isoforms. For doing so, we are required to develop novel methods or to 112 modify the existing statistical methods.

114 Our development work is based on Beta t-test of Baggerly et al (2003) (Baggerly et al. 115 2003)because this method is not sensitive to data distributions (see Discussion Section). 116 On the other hand, the Beta t-test approach optimizes weights for replicate libraries. The 117 weighting and optimal strategy may be useful for excluding artificial or technical noise in 118 count data and hence the genes or isoforms with better consistent counts in replicates 119 libraries but having differential transcriptions between conditions would be identified with 120 higher probabilities. The third, a very important point, is that it is t-test, a classical 121 distance-variance test approach, that is clear and simple to understand gene differential 122 expression but its fudge effect is also significant. For this reason, we are highly motivated to 123 develop a novel beta t-statistic by which gene mRNAs to be tested can be separated into 124 two different groups with least type I and type II errors.

\section{Statistical Methods}

128 Here we follow the notations of Baggerly et al (Baggerly et al. 2003) but for the 129 convenience of description, we use isoforms as features of study. However, our method is 
130

131

132

133

134

135

136

137

138

139

140

141 Considering the case of small sample size, we use weight to correct biases of expectation

142 and variance of estimated proportion $\mathrm{p}$. Supposing that we have $m$ replicate libraries in a

143 condition, the mean and variance of proportions in $m$ replicate libraries can be linearly

144 combined by weights (Baggerly et al. 2003):

145

$$
\begin{aligned}
& E\left(\sum_{i=1}^{m} w_{i} \hat{p}_{i}\right)=\sum_{i=1}^{m} w_{i} E\left(\hat{p}_{i}\right)=\frac{\alpha}{\alpha+\beta} \sum_{i=1}^{m} w_{i}=\frac{\alpha}{\alpha+\beta} \\
& V\left(\sum_{i=1}^{m} w_{i} \hat{p}_{i}\right)=\sum_{i=1}^{m} w_{i}^{2} V\left(\hat{p}_{i}\right)=\frac{w_{i}^{2} \alpha \beta}{(\alpha+\beta)(\alpha+\beta+1)}\left[\frac{1}{\alpha+\beta}+\frac{1}{N_{i}}\right]
\end{aligned}
$$


147 where the sum of weights over $m$ replicates is constrained to be 1 . Equation (2a) indicates

148 that this combination has the correct mean. Using a partial derivative of variance of 149 weighted proportions with respect to weights, solution for weight vectors can be 150 analytically given by

$$
w_{i} \propto\left[\frac{1}{\alpha+\beta}+\frac{1}{N_{i}}\right]^{-1} .
$$

152 Equation (3) indicates that the weights are determined by the means and sizes of libraries.

153 Here two extreme cases may occur: If $\alpha+\beta \rightarrow \infty$, then weight $w_{i}$ is proportional to size of

154 library $i, N_{i}$, meaning that distribution of $p_{i}$ is degenerate so that there is no change in the 155 true proportion going from sample to sample. If, on the other hand, $\alpha+\beta$ is very small, 156 then the weights would be roughly the same for all libraries. The true optimum lies 157 somewhat in between. With the weights, the proportion for an isoform count in a condition 158 is now estimated by

$$
\hat{p}=\sum_{i=1}^{m} w_{i} \hat{p}_{i}
$$

and its variance is also estimated, in an unbiased fashion, by

$$
\hat{V}^{*}=\frac{\sum_{i=1}^{m}\left(w_{i} \hat{p}_{i}\right)^{2}-\left(\sum_{i=1}^{m} w_{i}^{2}\right) \hat{p}^{2}}{1-\left(\sum_{i=1}^{m} w_{i}^{2}\right)} .
$$

162 Since we have weights for all parameters $(\alpha, \beta, p$, and $V)$ in a condition, then an iterative 163 search algorithm for optimal estimation of these parameters can be driven by weights (see 164 Supplemental Appendix B). 
166 Despite the estimation of variances of proportions in a condition is unbiased and 167 optimized, those isoforms of extremely small counts would have very small and similar 168 proportions in a few replicate libraries, which leads variances to be much smaller than 169 differences between means so that the $t$-values are inflated (see Discussion Section). To 170 avoid occurrence of this phenomenon, a modified estimator of variance is found to be

$$
\hat{V}=\max \left[\hat{V}^{*}, \hat{V}^{\#}\right] .
$$

172 In Baggerly et al(Baggerly et al. 2003), $\hat{V}^{\#}$ is given by

$$
\hat{V}^{\#}=\frac{\frac{\sum_{i=1}^{m} X_{i}}{\sum_{i=1}^{m} N_{i}}\left(1-\frac{\sum_{i=1}^{m} X_{i}}{\sum_{i=1}^{m} N_{i}}\right)}{\sum_{i=1}^{m} N_{i}} .
$$

174 From Equation(7a), we can find that when $\sum_{i=1}^{m} X_{i}=0$, then $\hat{V}^{\#}=0$. In addition, $\frac{\sum_{i=1}^{m} X_{i}}{\left(\sum_{i=1}^{m} N_{i}\right)^{2}}$

175 allows $\hat{V}^{\#}$ to be extremely small. In order to avoid the extreme small variance, we modify $176 \hat{V}^{\#}$ as

177

$$
\hat{V}^{\#}=\frac{\frac{1+\sum_{i=1}^{m} X_{i}}{\frac{1}{m} \sum_{i=1}^{m} N_{i}}\left(1-\frac{1+\sum_{i=1}^{m} X_{i}}{\frac{1}{m} \sum_{i=1}^{m} N_{i}}\right)}{\frac{1}{m} \sum_{i=1}^{m} N_{i}}
$$


$178 \hat{V}^{\#}$ in Equation (7b) is larger than that in Equation(7a). Equation (7b) shows that (1) lower 179 bound of $\hat{V}^{\#}$ is $\frac{m^{2}}{\left(\sum_{i=1}^{m} N_{i}\right)^{2}}\left(1-\frac{m}{\sum_{i=1}^{m} N_{i}}\right)$, not zero and (2) $\hat{V}^{\#}>\hat{V}^{*}$ when $\sum_{i=1}^{m} X_{i}$ is extremely 180 small. Thus $\hat{V}=\hat{V}^{\#}$ in case of extremely small $\hat{V}^{*}$.

By the above optimal estimation, we obtain $\hat{p}_{A}$ and $\hat{p}_{B}, \hat{V}_{A}$ and $\hat{V}_{B}$ in conditions $\mathrm{A}$ and $\mathrm{B}$, 183 respectively. Using these estimates, the t-statistic similar to $\mathrm{Z}$ statistic suggested by Kar et al (Kal et al. 1999) is found to be

$$
t=\frac{\hat{p}_{A}-\hat{p}_{B}}{\sqrt{\hat{V}_{A}+\hat{V}_{B}}}
$$

with degrees of freedom

$$
d f=\frac{\left(\hat{V}_{A}+\hat{V}_{B}\right)^{2}}{\frac{\hat{V}_{A}^{2}}{N_{A}-1}+\frac{\hat{V}_{B}^{2}}{N_{B}-1}}
$$

(Baggerly et al. 2003) where $N_{A}=\sum_{i=1}^{m_{A}} N_{A i}$ and $N_{B}=\sum_{i=1}^{m_{B}} N_{B i}$. With $d f$, we can obtain pvalue for each t-statistic from the t-distribution. For count-based transcriptional data, however, since replicate numbers are very small (for example, 3 replicate libraries), a 191 potential for "fudge" effect exists in a population. Although Equation (6) inserts another 192 estimator of variance as a lower bound so as to avoid occurrence of zero or extremely small 193 variance, the fudge effect still exists in Equation (8) due to small sample size. To remove 194 this effect, the t-statistic is modified as 


$$
t^{*}=\frac{\rho}{\omega} \frac{\hat{p}_{A}-\hat{p}_{B}}{\sqrt{\hat{V}_{A}+\hat{V}_{B}}} .
$$

196 Here $\rho$ is defined as $\rho=\sqrt{\phi \varphi}$ where $\psi$ is referred to as "polar ratio" (see Appendix C1). 197 Equation (C1) indicates that if two data sets $X_{A}=\left\{X_{A 1}, \cdots, X_{A m_{A}}\right.$ ) and $X_{B}=\left\{X_{B 1}, \cdots, X_{B m_{B}}\right.$ ) test(mBeta t-test) with setting $\rho=1$ and $\omega=1$ for all isoforms on the null simulated data,

214 find false DE isoforms, calculate their $\rho$ values, then order them from the smallest to the 
215 largest, $\rho_{1}<\rho_{2}<\cdots \rho_{j}<\cdots<\rho_{k}$, and calculate quantiles. We set $q_{1}=1 / k, q_{2}=2 / k, \cdots$,

$216 q_{j}=j / k, \cdots q_{k}=1$ where $\mathrm{k}$ is number of false discoveries in a null simulated data. Setting

$217 q_{j} \geq 0.85$, then we choose $\omega=\rho_{j}$ value. This means that $85 \%$ false discoveries would have

$218 \rho \leq \omega$ and be excluded. If, however, $\mathrm{k} \leq 5$, then $q_{j} \geq 0.85$ is not informative. In this case, we

219 take $\omega=\rho_{1}$. This process is done on all given simulated null datasets. We choose

$220 \bar{\omega}=\frac{1}{S} \sum_{s=1}^{S} \omega_{s}$ over S simulated null datasets. The p-value for each $\mathrm{t}^{*}$-value can be obtained

221 from t-distribution using degrees of freedom given or by performing the bootstrap (Storey

222 et al. 2005) (see Supplemental Appendix D).

\section{Simulation comparisons}

225 We used the 12 scenario stimulation datasets (see Simulation in Materials and Methods) to 226 compare 6 statistical methods for identifying isoforms differentially expressed between

227 two given conditions. The 6 statistical methods chosen here are Beta t-test (Baggerly et al.

228 2003), empirical Bayesian method (Hardcastle et al. 2010), Exact test (Robinson et al.

229 2010b; Robinson et al. 2007; Robinson et al. 2008), GLM (McCarthy et al. 2012), DESeq

230 (Anders et al. 2010)and our new Beta t-test (mBeta t-test). The empirical Bayesian

231 (eBayesian) method was implemented on R package baySeq and the Exact test and GLM

232 methods on R package edgeR(Robinson et al. 2010a). DESeq was implemented by R

233 package DESeq(Anders et al. 2010). The Beta and mBeta t-test methods were performed in

234 Matlab. We simulated null data to determine $\omega$ value for mBeta t-test. We set FDR cutoff =

2350.05 and chose estimated FDRs smaller than but closest to this cutoff as acceptable levels 
236 for differential expressions of isoforms because the FDR cutoff of 0.05 is widely accepted in 237 multiple tests, especially, in genome-wide studies. We counted isoforms identified to be 238 differentially expressed by these methods, false discoveries and calculated means and 239 standard deviations(stdev) of the numbers of findings and true FDRs for each method 240 chosen and then summarized them in Tables 1-3.

242 For small condition effect $(A=100)$ or low artificial noise proportion $(Q=10 \%)$, or low 243 proportion $(\mathrm{P}=10 \%)$ of $\mathrm{DE}$ isoforms, the Beta t-test method had higher power. Since mBeta 244 t-test is a modified beta t-test, in the case of low P or small A, mBeta t-test, eBaysian, Exact 245 test and GLM had similar powers, while in higher P and larger A scenarios, Beta and mBeta 246 t-test had lower powers than eBayesian, Exact test and GLM. In all 12 scenarios (Tables 1247 3), DESeq always had very low powers. This is because DESeq always had extremely low 248 true FDRs, indicating that DESeq is a very conservative method that would miss many true differentially expressed isoforms in practice. Beta t-test had much higher true FDRs than its 250 estimated FDRs in all these scenarios, meaning that in the Beta t-test method's findings, 251 there would be much more false discoveries than estimated, so this is not a conservative 252 method. eBayesian showed higher powers in low artificial noise proportion (Tables 1 and 253 3) but it also had higher true FDRs than estimated FDRs in most cases. In high artificial 254 noise proportion (Q=30\%) scenario, eBayesian performed well (Table 2). GLM showed 255 high powers in all 12 given scenarios but its true FDRs were much larger than estimated in 2569 scenarios (Table 1-3), suggesting that this method is also not conservative. In low DE 257 isoform proportion ( $\mathrm{P}=10)$, low artificial noise proportion $(\mathrm{Q}=10 \%)$ or small condition 
258 effect $(A=100)$ scenario, Exact test performed poorly because its true FDRs were much

259 larger than its estimated values in most cases, however, in high $\mathrm{P}(30 \%)$, high Q(30\%), and

260 large A(300) scenarios, Exact test had a good performance. Similarly to DESeq, mBeta t-

261 test also had lower true FDRs than its estimated values in all 12 given scenarios but its

262 powers were much higher than DESeq (Tables 1-3), showing that the mBeta t-test method

263 is conservative and powerful.

264

265 Stability is an important property of a statistical method. To rate stabilities of these

266 statistical methods in performance, we here used standard deviations (stdev) of finding

267 numbers and true FDRs listed in Tables 1-3 as criterion. Small stdev means that this

268 method has a small fluctuation and hence a high stability in identification of DE isoforms,

269 while larger stdev indicates that it has a bigger fluctuation and hence lower stability. Thus,

270 for each scenario, we ordered these methods by using stdev from the smallest to the

271 largest, assigned order scores (from 1 to 6 ) to them and averaged their order scores over

27212 scenarios. Thus, the order score can be used to measure relative stability of a method:

273 the smaller order score, the higher stability. Table 4 summarizes the results of stability

274 analysis. For findings, mBeta t-test had the highest stability, while GLM had the lowest.

275 Beta t-test, eBayesian, DESeq and Exact test got similar order scores and so they had

276 proximate stabilities. For true FDR, as expected, DESeq showed the highest stability. mBeta

277 t-test was in the second highest rank. GLM and Beta t-test were lowest. Exact test and 278 eBayesian showed similar stabilities. 
280 To comprehensively rate these statistical methods, we follow Tan (Tan 2011) and define work efficiency of a statistical method as

$$
w=\phi \varphi
$$

283 where $\phi=\frac{N_{f}}{N P}$ and $\varphi=1$ if true $\mathrm{FDR}<=0.05$ and $\varphi=0$, otherwise. Here $N$ is number of 284 isoforms in simulated data, $\mathrm{P}$, proportion of $\mathrm{DE}$ ioforms given in simulation, $N_{P}=N P$, and $285 N_{f}$, number of isoforms found to be differentially expressed by a statistical method. $\phi$ is 286 used to measure power (ability or probability) of a statistical method for identifying a 287 differentially expressed isoform and $\varphi$ to measure conservativeness of this method. The 288 performance of a method must be evaluated by its power and conservativeness. If a 289 method has a high power to find DE isoforms with no conservativeness, its findings would 290 then be unreliable and incredible; if a method has a low power with high conservativeness, 291 then the method would loss many findings. So such two types of statistical methods would 292 have low work efficiencies in identification of DE isoforms.

294 Table 5 lists work efficiencies of the 6 large-scale statistical methods in 4 pairs of scenarios. 295 From Table 5, one can find that eBayesian and GLM had higher work efficiencies in 3 296 replicate libraries than in 5 replicate libraries. This is because in 5 replicate libraries, the 297 two methods underestimated FDR at cutoff $\alpha=0.05$ (Tables 1-3) so that they lost 298 conservativeness. Beta t-test had work efficiency of zero in all scenarios. Exact test had 299 lower efficiencies in low $\mathrm{P}$, low $\mathrm{Q}$, small $\mathrm{A}$ and 3 replicates than in high $\mathrm{P}$, high $\mathrm{Q}$, large $\mathrm{A}$ 


\section{Real experimental data}

318 The simulated data are generally made in a known distribution and all factors impacting on

and 5 replicates. For the DESeq and mBeta t-test approaches, the work efficiency was greatly raised with increment of sample size. This is due to the fact that the two methods promoted its power with the same conservativeness. Similar results also can be seen in condition effects $A=100$ versus $A=300$. These two methods did not significantly respond to change in proportion of DE isoforms and in artificial noise proportion. However, in all simulated scenarios, the mBeta t-test method showed the highest work efficiencies.

In order to display FDR profiles along FDR cutoff, we here plotted averaged true FDRs against averaged estimated FDRs by these compared methods from cutoff $=\sim 0$ to $\sim 0.21$

in scenario 1. To evaluate the FDR profiles of these statistical methods, we also plotted a theoretical FDR profile (a diagonal line for true FDR against true FDR) for each method. Figure 1 shows that the estimated FDR curves of eBayesian, GLM and Exact test and Beta ttest are much below their theoretical lines, indicating that these methods, especially, Beta ttest, indeed heavily underestimated their FDRs, while DESeq too much overestimated its FDRs along the cutoff. Therefore, DESeq indeed is a too stringent and too conservative method. The mBeta t-test method overestimated FDR and hence is conservative. differential expressions are well controlled, hence evaluation of these statistical methods on the simulated data is conducted in ideal and known states. Obviously, such an evaluation 
321 has a limited significance for their application. Real data are therefore required in 322 comparison of statistical methods. However, since everything, in particular, noise 323 distribution in real data is unknown, a direct evaluation of statistical methods is impossibly 324 realized by comparison between true and estimated FDRs. For this reason, we here 325 employed an indirect way for doing so. First, we compared the performances of these 326 statistical methods on our two real datasets: Jurkat T-cell isoform transcriptomic data and 327 Jurkat T-cell gene transcriptomic data. The Jurkat T-cell isoform dataset contains 64428 328 isoforms which attribute to 14603 genes. These 64428 isoforms were annotated according 329 to alternative poly(A) and cleavage sites within genes and studied on differential 330 transcriptions between resting and stimulating immune states each with 3 replicate 331 libraries by using PAS-seq (Shepard et al. 2011). We used edgeR (Robinson et al. 2010a)to 332 normalize the Jurkat T-cell isoform and gene data. After filtering, 13409 isoforms in the 333 isoform data and 9572 genes in the gene data were available for differential expression 334 analyses. eBayesian had no results because either it was running too long (over at least 2 335 days in isoform data, infinite loop might occur) or showed NA result (in gene data). GLM 336 obtained 4376 genes and 5039 isoforms of being differentially expressed from our gene 337 and isoform data, respectively, at FDR cutoff of 0.05 . Since ratios of findings are so high 338 (45\% in the gene data and $37 \%$ in the isoform data), we did not believe that this method 339 could work on these real data. DESeq found $261 \mathrm{DE}$ genes and $287 \mathrm{DE}$ isoforms at the same 340 FDR cutoff. The ratios of findings are so low ( $3 \%$ in gene data and $2 \%$ in isoform data), 341 basically, DESeq also did not work on the two transcriptomic data. The Beta t-test, mBeta t342 test and Exact test methods worked and the results obtained at FDR cutoff of 0.05 are listed 343 in Table 6. 
345 In the next step, we compared their findings using Venn Diagram Generator 346 (http://simbioinf.com/mcbc/applications/genevenn/). Figure 2A shows that except that 347 the three methods shared 554 DE genes, Exact test and mBeta t-test shared 22 DE genes in 348 their 2019 findings while the former and Beta t-test shared merely 3 DE genes in their 3492760 findings. Similar result was also found in the isoform data (Fig. 2B). In addition, if a 350 gene was found to be differentially expressed by a method only, then it is highly possible 351 that this DE gene would be falsely discovered. Figure 3 visualizes heat maps of 10 genes 352 identified to be differentially expressed by mBeta t-test method (Fig. 3A), 770 by Beta t-test 353 only (Fig. 3B), and 220 by Exact test only (Fig. 3c). Indeed, the method-specific genes do not 354 display obvious expression difference between no stimulation (NS) and 48h 355 poststimulation. Thus, we defined no share ratio of findings $\left(m_{i} / M_{i}\right.$ where $m_{i}$ is number of 356 method $i$-specific findings, and $M_{i}$ is numbers of findings identified by method $i$ ) as least 357 true false discovery rate (least true FDR is corresponding to q-value defined by Storey et 358 (Storey et al. 2003)). Using this indirect method, we obtained the least true FDRs for the 359 findings of the Exact test, mBeta t-test and Beta t-test methods, respectively, in the two real 360 transcriptomic datasets (Table 6). From Table 6, one can see that Exact test and Beta t-test 361 extremely underestimated their FDRs while mBeta t-test still overestimated FDR in its 362 findings. These are well consistent with those found in the simulated data.

\section{Experimental Validation}


365 qRT-PCR experiments were carried out to valid our method's findings. Our qRT-PCR 366 experiments were done in Jurkat T-cells at rest and 48 hours after stimulation. To make 367 our qRT-PCR results be more representative, we randomly chose the genes that were found 368 to be up-regulated or down-regulated at 48 hours after stimulation and not to be 369 differentially expressed by mBeta t-test. We used relative differences between stimulation 370 and rest and relative variation coefficient (VC) (see Materials and Methods Section) to do 371 comparison between the RNA-seq and qRT-PCR data. The results show that genes UBL3, 372 MST123 and KIAA0465 that were found to be up-regulated to respond to stimulation (blue 373 columns in Fig. 4A) in RNA-seq data also displayed positive response to stimulation in qRT374 PCR data (red columns in Fig. 4A). Gene CD47 negatively responded to stimulation in both 375 datasets while gene TESK2 was not detected to have significantly difference between 376 stimulation and rest in these two datasets. In expression direction and relative expression 377 amount, these two datasets show cc=0.9 (Pearson correlation coefficient) (Fig. 4 A), 378 suggesting that our transcriptomic data were well consistent with qRT-PCR data. Using 379 relative $\mathrm{VC}$, we found that gene UBL3 had small expression noise at rest and stimulation in 380 these two datasets, while genes TESK2 had bigger expression noises in the transcriptomic 381 data (Fig. 4 B). This is why gene UBL3 was detected to be differentially expressed but 382 TESK2 was not though both UBL3 and TESK2 had small counts of mRNA reads in 383 transcriptomic data. 
Although our simulated data were made in the NB distributions, Beta and mBeta t-test worked well if estimation of FDR was not considered. This is because not only the NB counts can be well approximated by binomial distributions but also the frequencies (p) of counts of mRNA reads in libraries can be approximated by beta distributions. While the eBayesian, GLM, Exact test, and DESeq approaches are merely based on the NB distribution. Therefore, for real datasets whose distributions are often unknown, mBeta t-test will perform well. For example, as seen in Result Section, eBayesian and GLM, DESeq could not work on our Jurkat T-cell isoform and gene data, while Exact test, Beta t-test and mBeta ttest worked even though their results have big differences. We also applied these methods to our leukemia transcriptomic data containing 10299 genes (data not yet published), the results show that all methods chosen can work but eBayesian has very low power (it just found 165 DE genes), while GLM, Exact test and mBeta t-test identified, respectively, 780, 733 and 711 DE genes and hence performed very similarly. These strongly suggest that eBayesian and GLM are specific to the NB distribution.

In addition, in genome-wide data, especially, in transcriptomic data, sample sizes usually are very small, for example, 3 or 2 replicate libraries in each condition due to high cost and biological resource limitation. Small samples would lead to a fudge effect (Tan 2011; Tan et al. 2011; Tan et al. 2006). For example, in count data containing more than ten thousands of isoforms, two 3 -replicate small-count sets would have a larger probability of showing that they would be sampled from the same distribution not only than two 5-replicate smallcount sets but also than two 3-replicate big-count sets. On the other hand, in 
408 transcriptome-wide data, small-count data have more chance to be weakly fluctuated by 409 noise and to form extremely small within-variances than big-count data, giving rise to 410 inflating t-statistics. For general statistical methods, the genes or isoforms with small count 411 data in small samples would easily be found to be differentially expressed between 412 conditions due to inflation of statistic. To address this problem, many methods developed 413 for identifying differentially expressed genes in microarray data introduce a constant to 414 shrink statistics. For example, In SAM (Tusher et al. 2001), two-sample t-test is modified as 415 S-test by adding a minimized coefficient of variation $\mathrm{S}_{0}$ of differences between two 416 conditions to denominator. In the regularized $t$ test (Baldi et al. 2001), two-sample t-test is 417 modified by combining gene-specific variance with global average variance. The two 418 methods shrink all t-statistics across the whole genes detected in microarrays. So they have 419 low powers (Tan 2011). Tan et al (Tan et al. 2006) used a conditional shrinking method to 420 address the problem of inflating t-test. But this conditional shrinking method cannot be 421 introduced to Beta t-test because the Beta t-test is based on differences in frequencies 422 (proportions) of tags between conditions (Baggerly et al. 2003).

424 Baggerly et al (Baggerly et al. 2003) employed a weighting and iteration strategy to look for 425 an optimal estimation of parameters beta and alpha of frequency that is assumed to follow 426 beta distribution for a tag in a condition and furthermore developed a new t-test, we called 427 Beta t-test. Weight and optimization is a strategy for excluding artificial or technical noise 428 in count data. Although Baggerly et al (Baggerly et al. 2003) recognized small-count effect 429 on t-tests and tried to avoid the problem of t-value inflation using alternative variance 
430 given in Equation (18a), our practice demonstrated that Equation (18a) does not 431 substantially reduce the fudge effect. For this reason, we modify the alternative variances 432 by utilizing means of total counts over all libraries in a condition for those isoforms with 433 very small counts. Analytically, it can be seen that the alternative variance defined in 434 Equation (18b) is larger than that in Equation (18a). Our simulation really showed that the 435 above small-count effect on testing for differential expressions of isoforms was mostly 436 reduced by our modified alternative variances.

438 In order to eliminate small sample effect, we introduced a gene-or isoform-specific variable $439 \rho$ into the Baggerly et al.s' (Baggerly et al. 2003)Beta t-test. $\rho$ is used to measure overlap 440 between two count sets. If two count sets more overlap, then $\rho$ becomes smaller; if two 441 count sets separate, then $\rho>1$. The larger gap between two count sets, the larger $\rho$. In 442 theory, two count sets that are separated have a higher probability of showing that they 443 came from two different populations than those that overlap. Besides, if noise within count 444 sets is large, then $\zeta$ is small, which makes $\rho$ become small. Thus, $\rho$ shrinks t-values of 445 overlapped count sets and inflates t-values of separated count sets with small noise. As 446 seen in Tables 1-3, compared to the Beta t-test method, our mBeta t-test approach did not 447 obviously decrease its power but significantly reduce false discovery rate so that it has 448 higher work efficiencies. Considering sample size effect, we set a threshold $\omega$ for $\rho$. That 449 is, t-values are inflated with $\rho>\omega$, or shrunken with $\rho<\omega$. As a result, almost all of small 450 t-values are compressed into a short interval close to zero but the t-values with $\rho>\omega$ are 451 further enlarged and a region in which truly differential expressions of isoforms and 
452 expression noises are mixedly distributed becomes very narrow(Fig. 5). Since p-value only

453 depends on t-value given degree of freedom, $\mathrm{p}$-values with inflating t-values become 454 smaller while those with shrinking t-values become larger. Thus a lot of false discoveries 455 are also compressed into the zero neighbors so that very few false positives would be 456 found (Fig.5). Threshold $\omega$ depends on sample size. The larger sample size, the smaller $\omega$. 457 However, when sample size is large, $\omega$ becomes very small, ability of $\rho$ controlling false 458 discoveries becomes very weak because the gaps between two datasets are vanished and 459 there is not fudge effect in such data.

461 ROC is popularly used to evaluate statistical methods (Hardcastle et al. 2010) (Robinson et 462 al. 2007) but ROC has two fatal drawbacks. First, ROC cannot evaluate the FDR estimation. 463 For multiple tests, since FDRs are unknown, they must be estimated so that one can 464 determine which features have statistical significances. Precise or conservative estimation 465 of FDRs is important for an experimental scientist or statistician to choose a statistical 466 method in practice because if a method significantly underestimates FDRs in findings, then 467 it would provide much more false findings than expected or if it much overestimates FDRs, 468 we would then loss many true findings (Tan 2011). Second, in some cases, the R0C curves 469 of the methods are tightly close to each other or overlap together, meaning that these 470 methods have similar sensitivity against specificity but it does not suggest that they have 471 the same or approximate performances because they may have different estimations of 472 FDRs. For example, we employed simulated data of scenarios 1 and 4 to makeup 473 eBayesian, Exact test, DESeq and mBeta t-test ROC curves. The results show that mBeta t- 
474 test performed best at specificity< $<0.3$ in scenario 1 (Fig. 6A) or $<0.5$ in scenario 4 (Fig. 6B),

475 DESeq had the slight higher sensitivity than mBeta t-test when specificity $>0.3$ in scenario

4761 (Fig. $6 \mathrm{~A}$ ) or $>0.5$ in scenario 4 (Fig. 6B), eBayesian had the lowest curve, Exact test and

477 GLM had almost the same curve, performed better than eBayesian when specificity $>0.4$

478 (Fig. 6). However, these ROC curves did not show significant difference, in particularly, in 479 scenario 4. For this reason, our evaluation of these methods chosen was based on 480 comparison between true and estimated FDRs. The simulated data showed that the 481 eBayesian and GLM methods worked well in the ideal NB distributions, lower proportions 482 of DE isoforms, smaller condition effect and smaller number of replicate libraries but they 483 performed poorly in the case in which sample sizes were larger and proportion of DE 484 isoform was higher or condition effect was bigger. This is because in such a scenario they 485 generally had a high power to find DE isoforms but, on the other hand, their FDRs are often 486 notably underestimated. Therefore, we evaluated performance of a statistical method in 487 power (ability or probability to find DE genes or isoforms) and conservativeness of FDR 488 estimation (reliability of findings). A statistical method with high power but no 489 conservativeness of FDR estimation would offer us a lot of unreliable findings or a 490 statistical method with low power but conservativeness would miss many true DE genes or 491 isoforms. That is to say, these two types of methods would have low work efficiency to 492 perform their statistical analysis of real data.

494 It is required to indicate that the Exact test method performs very fast, in contrast, the 495 eBayesian method (baySeq) is very intensive in computation and took a long time (maybe 
496 infinite loop occurred in calculation of posterior probabilities when performed on our real 497 data). Nevertheless, the current version of baySeq is not available for our real data. The 498 mBeta t-test method is also computationally intensive because it runs literately to look for 499 an optimal estimation of weight and beta and alpha parameters for each isoform. However, 500 it finishes its work in 15 minutes for more 10 thousands of isoforms if we don't use 501 bootstrap to calculate p-values.

\section{Cell Lines and Stimulation}

Jurkat T-cell lines were obtained from the ATCC and maintained in RPMI (ATCC) with 10\%

506 fetal bovine serum supplemented with penicillin and streptomycin (Gibco). Jurkat T-cells

507 were stimulated with plate-bound antibodies coated with a solution of 1 ?g anti-CD3

508 (OKT3 - eBiosciences) and 5 ? g anti-CD28 (CD28.2 - BD Pharmingen). Activation of T-cell 509 was monitored via flow cytometry detection of CD69 expression (FN-50) 48 hours after 510 stimulation (Simms et al. 1996).

\section{High-throughput Sequencing Library Generation}

513 Total RNA was harvested from resting and stimulated cells with Trizol reagent and 514 processed as per manufacturer instructions. Polyadenylated RNA was isolated with the 515 Poly(A)-Purist MAG kit as per manufacturer instructions. Libraries for high-throughput 
516 sequencing were established as described (Shepard et al. 2011) with minor modifications.

517 Libraries were sequenced via 50 bp paired-end sequencing on an Illumina GAIIx in genome 518 sequencing center in Baylor College of Medicine. The listed reagents were from Life 519 Technologies.

\section{Annotation and pipeline analyses}

522 Paired-end reads were first subjected to a profiler removing $\mathrm{A}$ and $\mathrm{T}$ homopolymer runs

523 within the forward and reverse reads, respectively. Pairs in which the length of both reads

524 was greater than 25 bp were mapped to the human genome reference (UCSC hg19) with

525 Bowtie(Langmead et al. 2009) using default parameters. The reads were simultaneously

526 mapped to the UCSC KnownGene database to identify putative exon-spanning reads or 527 pairs. The union of mate pairs mapping uniquely to the genome and those mapping 528 specifically to the KnownGene database were condensed to isoforms by 3' alignment 529 identity, filtered for false priming, and assigned gene identity and region (e.g. CDS, 3' UTR) 530 using UCSC KnownGene annotations. Cleavage and polyadenylation sites were defined as 531 the median genome coordinate of all reads within a 20-base pair sliding window of an

532 adjacent read. Defined sites were carried forward for analysis only if they were present in 533 all libraries representing an individual cellular type or state. Intralibrary isoform 534 representation was normalized to pseudocounts using the negative binomial 535 method(Robinson et al. 2008). 


\section{7 qRT-PCR}

538 Total RNA was respectively isolated from Jurkat T-cells at rest and 48hours after stimulation using

539 TRIZOL(Invitrogen). RNA was digested with DNase I to remove contaminating genomic DNA.

540 One microgram of total RNA was used to generate cDNA with the ImProm-II ${ }^{\mathrm{TM}}$ Reverse

541 Transcription System (Promega) and real-time PCR was performed in triplicates in an

542 Eppendorf Mastercycler. qRT-PCR was performed using a pair of primers(forward and

543 reverse primers) designed for a selected gene. $\Delta \Delta \mathrm{C}_{\mathrm{T}}$ method was used to calculate relative

544 quantitation of qRT-PCR product on a 7500 Fast Real-Time PCR machine with SDS software

545 (Applied Biosystems).

547 Simulations

548 To evaluate statistical properties of various approaches, we used the NB pseudorandom 549 generator to create RNA isoform count datasets in 12 scenarios, each repeated three times 550 for calculations of means and standard deviations. Our simulations were conducted on our

551 Jurkat T-cell isoform data from which we took 18290 isoforms and 3 replicated libraries in 552 each of two conditions (resting and stimulating states). We set two levels ( $A=100$ and 300$)$ 553 of condition (or treatment) effect on differential transcription of isoforms and linearly 554 assigned the effects $\tau=U A$ to differentially expressed isoforms where $\mathrm{U}$ is uniform variable $555(U \in(0,1])$, two levels of proportions of differentially expressed isoforms: $\mathrm{P}=10$ and $30 \%$, 556 two levels of artificial noise proportions: $Q=10$ and $30 \%$ and 2 levels of sample sizes: $R=3$ 557 and 5 replicate libraries. Here artificial noise (also called technique or Poisson noise) 558 indicates that the noise does not comes from experimental system error but come from 
559 techniques such as sequencing, mapping, annotation and pipline analysis, etc. In simulated 560 data, isoforms with averaged count $<5$ were filtered, thus 18162 isoforms were available for 561 analysis.

\section{Software}

564

565

566

567

568

569

570

571

572

573

574

575

576

577

578

579

580

581

A package for implementing mBeta t-test was written in Matlab and a program for generating simulation data was written in R. They are available for request.

\section{Acknowledgement}

We are indebted to Natee Kongchan for library preparation and Alexander Ruch and Jixin Deng for primary processing of our data sets. This work was funded by The Cancer Prevention and Research Institute of Texas (HIRP100475) and the National Cancer Institute (CA126752, CA131474).

\section{References}

Anders, S. and Huber, W. 2010. Differential expression analysis for sequence count data. Genome Biol 11: R106.

Baggerly, K.A., Deng, L., Morris, J.S., and Aldaz, C.M. 2003. Differential expression in SAGE: accounting for normal between-library variation. Bioinformatics 19: 1477-1483.

Baggerly, K.A., Deng, L., Morris, J.S., and Aldaz, C.M. 2004. Overdispersed logistic regression for SAGE: modelling multiple groups and covariates. BMC Bioinformatics 5: 144.

Baldi, P. and Long, A.D. 2001. A Bayesian framework for the analysis of microarray expression data: regularized t -test and statistical inferences of gene changes. Bioinformatics 17: 509-519. 
Cloonan, N., Forrest, A.R., Kolle, G., Gardiner, B.B., Faulkner, G.J., Brown, M.K., Taylor, D.F., Steptoe, A.L., Wani, S., Bethel, G. et al. 2008a. Stem cell transcriptome profiling via massive-scale mRNA sequencing. Nat Methods 5: 613-619.

Cloonan, N. and Grimmond, S.M. 2008b. Transcriptome content and dynamics at single-nucleotide resolution. Genome Biol 9: 234.

Degner, J.F., Marioni, J.C., Pai, A.A., Pickrell, J.K., Nkadori, E., Gilad, Y., and Pritchard, J.K. 2009. Effect of read-mapping biases on detecting allele-specific expression from RNA-sequencing data. Bioinformatics 25: 3207-3212.

Denoeud, F., Aury, J.M., Da Silva, C., Noel, B., Rogier, O., Delledonne, M., Morgante, M., Valle, G., Wincker, P., Scarpelli, C. et al. 2008. Annotating genomes with massive-scale RNA sequencing. Genome Biol 9: R175.

Hardcastle, T.J. and Kelly, K.A. 2010. baySeq: empirical Bayesian methods for identifying differential expression in sequence count data. BMC Bioinformatics 11: 422.

Kal, A.J., van Zonneveld, A.J., Benes, V., van den Berg, M., Koerkamp, M.G., Albermann, K., Strack, N., Ruijter, J.M., Richter, A., Dujon, B. et al. 1999. Dynamics of gene expression revealed by comparison of serial analysis of gene expression transcript profiles from yeast grown on two different carbon sources. Mol Biol Cell 10: 1859-1872.

Langmead, B., Trapnell, C., Pop, M., and Salzberg, S.L. 2009. Ultrafast and memory-efficient alignment of short DNA sequences to the human genome. Genome Biol 10: R25.

Li, J., Jiang, H., and Wong, W.H. 2010. Modeling non-uniformity in short-read rates in RNA-Seq data. Genome Biol 11: R50.

Madden, S.L., Galella, E.A., Zhu, J., Bertelsen, A.H., and Beaudry, G.A. 1997. SAGE transcript profiles for p53-dependent growth regulation. Oncogene 15: 1079-1085.

Man, M.Z., Wang, X., and Wang, Y. 2000. POWER_SAGE: comparing statistical tests for SAGE experiments. Bioinformatics 16: 953-959.

McCarthy, D.J., Chen, Y., and Smyth, G.K. 2012. Differential expression analysis of multifactor RNA-Seq experiments with respect to biological variation. Nucleic Acids Res 40: 4288-4297.

Michiels, E.M., Oussoren, E., Van Groenigen, M., Pauws, E., Bossuyt, P.M., Voute, P.A., and Baas, F. 1999. Genes differentially expressed in medulloblastoma and fetal brain. Physiol Genomics 1: 83-91.

Montgomery, S.B., Sammeth, M., Gutierrez-Arcelus, M., Lach, R.P., Ingle, C., Nisbett, J., Guigo, R., and Dermitzakis, E.T. 2010. Transcriptome genetics using second generation sequencing in a Caucasian population. Nature 464: 773-777.

Morrissy, A.S., Morin, R.D., Delaney, A., Zeng, T., McDonald, H., Jones, S., Zhao, Y., Hirst, M., and Marra, M.A. 2009. Next-generation tag sequencing for cancer gene expression profiling. Genome Res 19: $1825-1835$.

Mortazavi, A., Williams, B.A., McCue, K., Schaeffer, L., and Wold, B. 2008. Mapping and quantifying mammalian transcriptomes by RNA-Seq. Nat Methods 5: 621-628.

Pan, Q., Shai, O., Lee, L.J., Frey, B.J., and Blencowe, B.J. 2008. Deep surveying of alternative splicing complexity in the human transcriptome by high-throughput sequencing. Nat Genet 40: 14131415.

Robinson, M.D., McCarthy, D.J., and Smyth, G.K. 2010a. edgeR: a Bioconductor package for differential expression analysis of digital gene expression data. Bioinformatics 26: 139-140.

Robinson, M.D. and Oshlack, A. 2010b. A scaling normalization method for differential expression analysis of RNA-seq data. Genome Biol 11: R25.

Robinson, M.D. and Smyth, G.K. 2007. Moderated statistical tests for assessing differences in tag abundance. Bioinformatics 23: 2881-2887.

Robinson, M.D. and Smyth, G.K. 2008. Small-sample estimation of negative binomial dispersion, with applications to SAGE data. Biostatistics 9: 321-332. 
Shepard, P.J., Choi, E.A., Lu, J., Flanagan, L.A., Hertel, K.J., and Shi, Y. 2011. Complex and dynamic landscape of RNA polyadenylation revealed by PAS-Seq. RNA 17: 761-772.

Simms, P.E. and Ellis, T.M. 1996. Utility of flow cytometric detection of CD69 expression as a rapid method for determining poly- and oligoclonal lymphocyte activation. Clin Diagn Lab Immunol 3: 301-304.

Storey, J.D. and Tibshirani, R. 2003. Statistical significance for genomewide studies. Proc Natl Acad Sci U S A 100: 9440-9445.

Storey, J.D., Xiao, W., Leek, J.T., Tompkins, R.G., and Davis, R.W. 2005. Significance analysis of time course microarray experiments. Proc Natl Acad Sci U S A 102: 12837-12842.

t Hoen, P.A., Ariyurek, Y., Thygesen, H.H., Vreugdenhil, E., Vossen, R.H., de Menezes, R.X., Boer, J.M., van Ommen, G.J., and den Dunnen, J.T. 2008. Deep sequencing-based expression analysis shows major advances in robustness, resolution and inter-lab portability over five microarray platforms. Nucleic Acids Res 36: e141.

Tan, Y.D. 2011. Work efficiency: A new criterion for comprehensive comparison and evaluation of statistical methods in large-scale identification of differentially expressed genes. Genomics 98: 390-399.

Tan, Y.D. and Fornage, M. 2011. Effects of genetic and environmental factors and gene-environment interaction on expression variations of genes related to stroke in rat brain. American Journal of Molecular Biology 1: 87-113

Tan, Y.D., Fornage, M., and Fu, Y.X. 2006. Ranking analysis of microarray data: a powerful method for identifying differentially expressed genes. Genomics 88: 846-854.

Tusher, V.G., Tibshirani, R., and Chu, G. 2001. Significance analysis of microarrays applied to the ionizing radiation response. Proc Natl Acad Sci U S A 98: 5116-5121.

Wang, L., Feng, Z., Wang, X., and Zhang, X. 2010. DEGseq: an R package for identifying differentially expressed genes from RNA-seq data. Bioinformatics 26: 136-138.

Wang, Z., Gerstein, M., and Snyder, M. 2009. RNA-Seq: a revolutionary tool for transcriptomics. Nat Rev Genet 10: 57-63.

Wu, Z.J., Meyer, C.A., Choudhury, S., Shipitsin, M., Maruyama, R., Bessarabova, M., Nikolskaya, T., Sukumar, S., Schwartzman, A., Liu, J.S. et al. 2010. Gene expression profiling of human breast tissue samples using SAGE-Seq. Genome Res 20: 1730-1739.

Zhang, L., Zhou, W., Velculescu, V.E., Kern, S.E., Hruban, R.H., Hamilton, S.R., Vogelstein, B., and Kinzler, K.W. 1997. Gene expression profiles in normal and cancer cells. Science 276: 1268-1272.

\section{Figure legends}

\section{Figure 1 Profiles of true versus estimated FDR}

Estimated curve was made by plotting estimated FDR against true FDR along cutoff of $\sim 0$ to $\sim 0.21$ and theoretical line is a diagonal line made by plotting true FDR against true FDR along the same cutoff. The true FDR was calculated by counting false positives in findings of a statistical method at an FDR cutoff point in a 
670

671

672

673

674

675

676

677

678

679

680

681

682

683

684

685

686

687

688

689

690

691

692

693

694

695

696

697

698

simulated dataset and the estimated FDR was predicted by a statistical method. The true and estimated FDRs given figure 1 were averaged over three simulated datasets in scenario 1.

Figure 2 Venn diagram analyses of the Exact test, Beta t-test and mBeta t-test approaches

A: DE genes found in Jurkat T-cell gene transcriptomic data. B: DE isoforms found in Jurkat T-cell isoform transcriptomic data.

Figure 3 Heatmaps of method-specific differentially expressed genes

NS.A, NS.B, NS.C are replicate libraries A, B and C in resting state (no stimulation). 48h.A, 48h.B and 48h.C are replicate libraries A, B and C in stimulating state (48h poststimulation). Method-specific DE genes were treated as false DE genes. Heatmaps show that these genes really do not have obvious expression difference between resting and stimulating states. A: $10 \mathrm{DE}$ genes found by mBeta t-test only. B: $770 \mathrm{DE}$ genes found by Beta test only. C: 220 DE genes found by Exact test only.

Figure 4 Comparison between RNA-seq data and qRT-PCR data on 5 genes chosen.

In RNA-seq data genes UBL3, MST123, CD47 and KIAA0465 were found to be differentially expressed between rest and stimulation. TESK2 had no differential expression. Genes UBL3, MST123, KIAA0465 were significantly higher at stimulation than at rest, while genes CD47 and BCL11B were significantly lower at stimulation than at rest.

A: Relative expression comparisons of 5 genes between RNA-seq and qRT-PCR. In RNA-Seq data, relative expression of a gene is defined as $d_{g} / \bar{d}$ where $d_{g}=\bar{n}_{g t}-\bar{n}_{g 0}, \bar{d}$ is averaged value, g= UBL3, MST123, CD47, KIAA0465 and TESK2, $\bar{n}$ is averaged count of reads over three replicates and $\mathrm{t}=48$ hour. In qRT-PCR, the relative expression of a gene is defined as $\Delta_{g}-\Delta_{b}$ where $\Delta_{g}=\overline{C T}_{g t}-\overline{C T}_{g 0}, \mathrm{~b}=$ background gene for control, and $\overline{C T}$ is averaged CT value over three replicates and CT is $\log 2$ transformed threshold value of amplification in qRT-PCR. In our experiment, we used TBP (TATA binding protein) as background expression because it has no change in expression with time.

B: Relative expression variation coefficient comparison of 5 genes between RNA-seq and qPCR data. Relative expression variation coefficient is defined as $V C_{g t} / \overline{V C}_{t}$ where $V C_{g t}=\bar{n}_{g t} / s_{g t}$ and $\overline{V C}_{t}$ is averaged variation coefficient over all selected genes at time $t$ of stimulation.

\section{Figure 5 Plots of t-statistics versus $\log \mathrm{FC}$.}

$\operatorname{logFC}=\log$ (mean in $48 \mathrm{~h}$ poststimulation/mean in NS) and t-values were given by two beta t-test methods from the simulated data in which $10 \%$ of isoforms randomly assigned with condition effect $\tau \leq 100$ were 
differentially expressed between two conditions each with three replicate libraries. Simulation was conducted by NB pseudorandom generator based on real isoform transcriptomic data.

A The Beta t-statistics are distributed in interval between -16 and 16, while those for no differential expressed genes in Beta t-test are distributed in interval between -4.8 and 4.8. Thus a lot of false discoveries (blue dots) are distributed in the neighboring areas of $t$-value $\geq 4.8$ or $t$-value $\leq-4.8$.

B: Plots of the mBeta t-statistics versus log FC. The t-statistic interval is enlarged from below -50 to over 100, while the t-statistics for no differential expressed genes are strongly compressed into a very narrow area close to zero. Thus a lot of false discoveries of the Beta t-test method are also moved into this area so that very few false positives (blue dots) would be found.

\section{Figure 6 ROC comparison of statistical methods}

ROC curves of the eBayesian, Exact test, GLM, DESeq and mBeta t-test methods were made from simulated datasets. Sensitivity $=$ true positive fraction (TPF) and specificity $=$ false positive fraction(FPF). A: simulated data came from scenario 1 (proportion of differentially expressed isoforms $=10 \%$, technical noise proportion= $10 \%$ and treatment effect $A=100$, sample size $=3$ ) and $B$ : from scenario 4 (proportion of differentially expressed isoforms $=30 \%$, technical noise proportion $=10 \%$ and treatment effect $A=300$, sample size $=3$ ) . 4 (1)

(1)

(1)



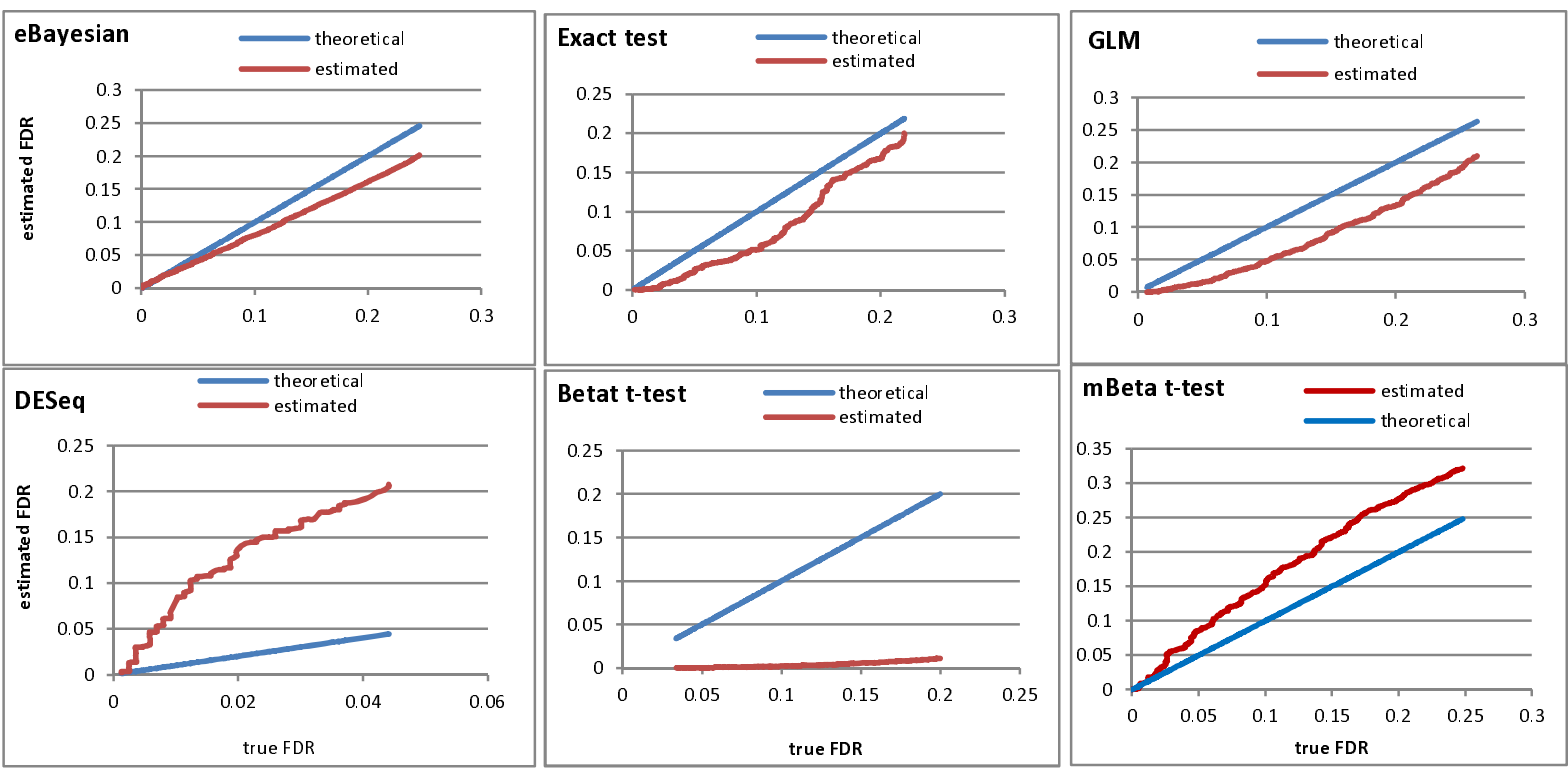

731

732

733

734

735

736

A

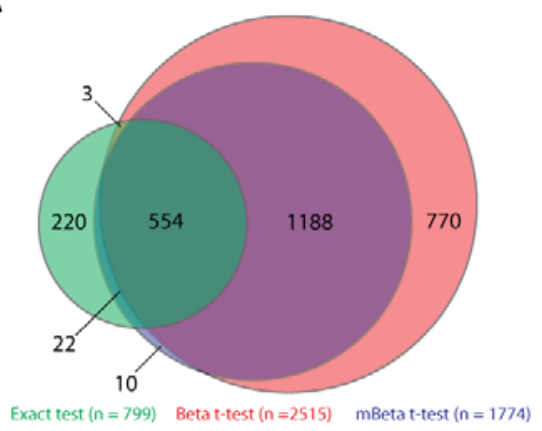

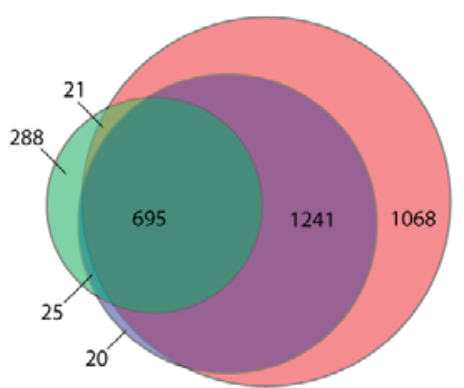

Exact test $(n=1029) \quad$ Beta t-test $(n=3025) \quad$ mBeta t-test $(n=198$

737 

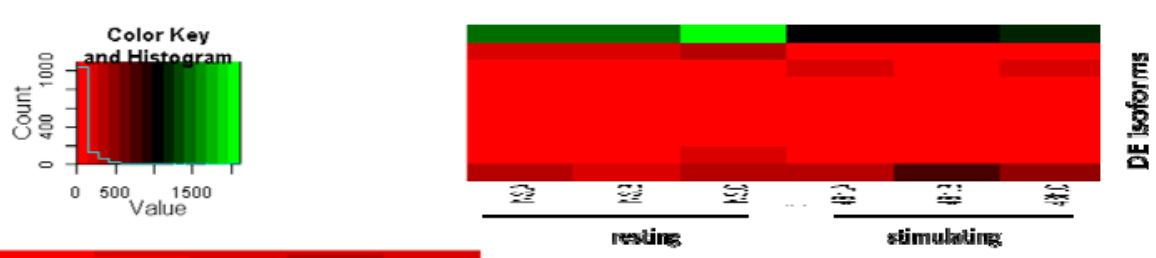

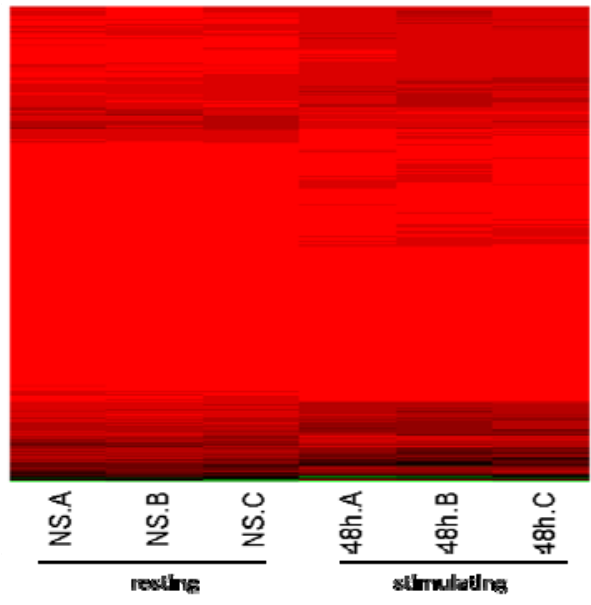

B

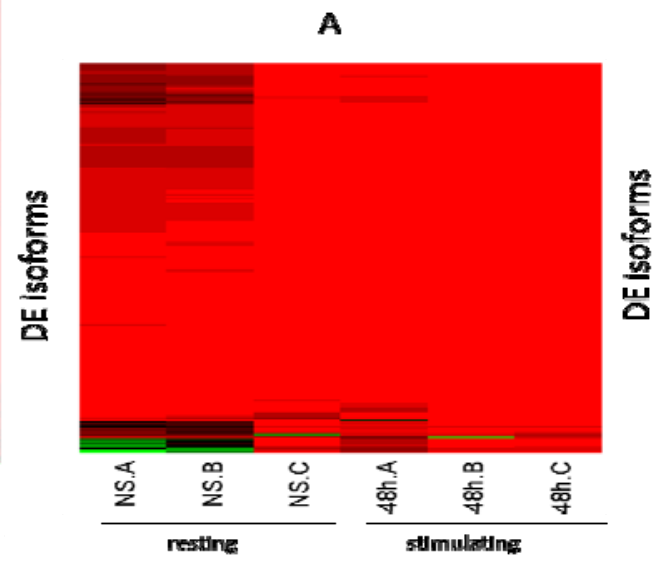

c
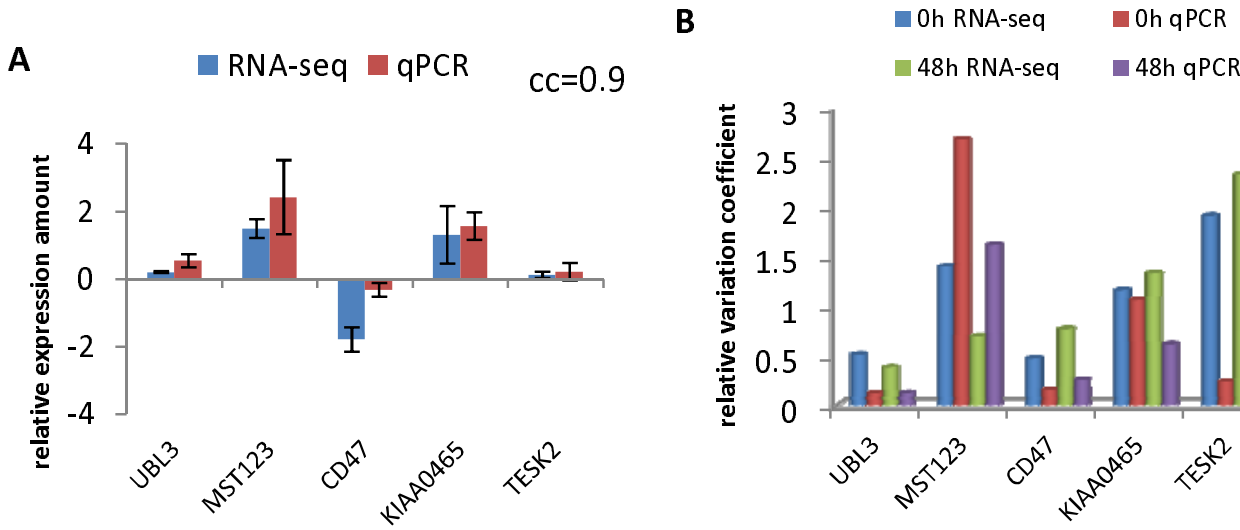
751

752

753

754

755

756

757

758

759

760

761

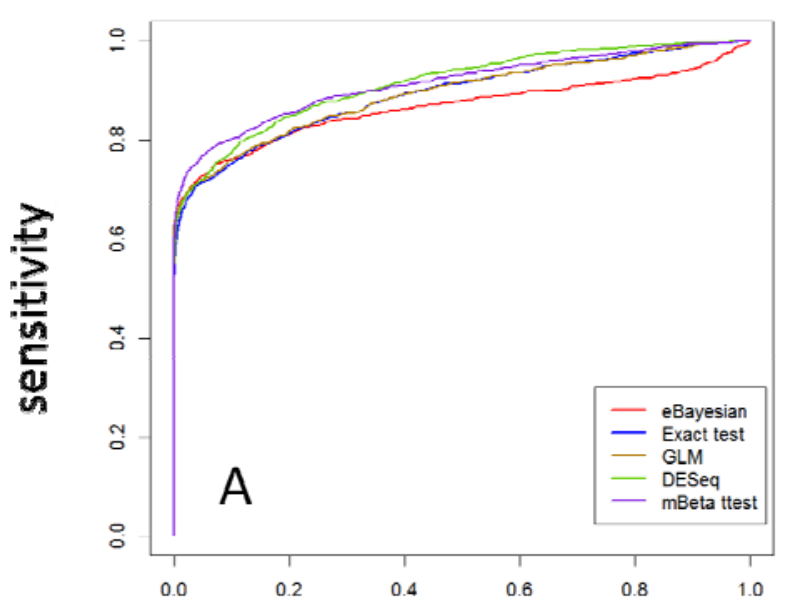

specificity

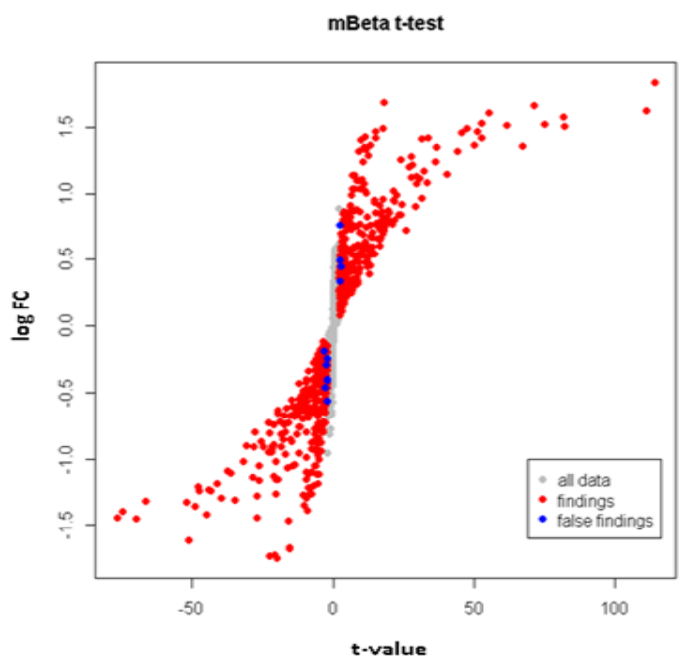

Figure 5

763

Figure 6

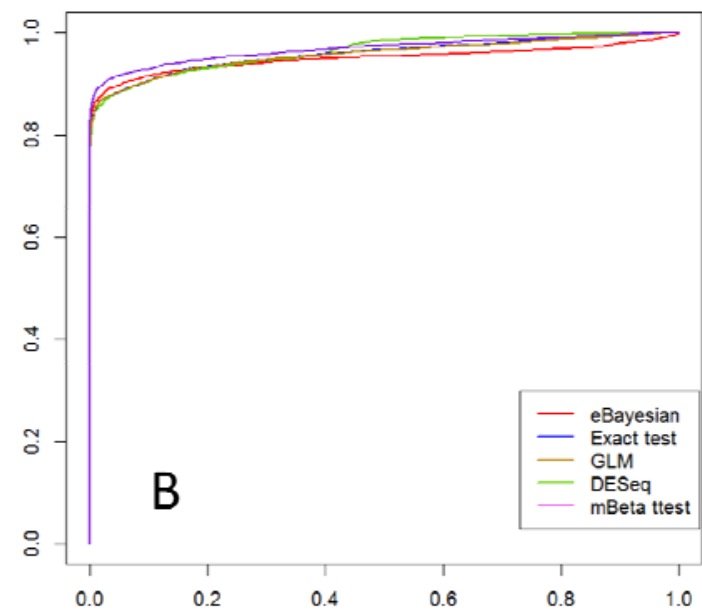

\section{specificity}


Table 1 Results of performing statistical methods on simulated data of 18162 isoforms and two conditions in simulation scenarios 1 - 4 where simulations were based on real transcriptomic data

\begin{tabular}{|c|c|c|c|c|c|c|}
\hline \multirow[t]{2}{*}{ scenario } & \multirow{2}{*}{$\begin{array}{l}\text { Statistical } \\
\text { Method }\end{array}$} & \multicolumn{2}{|c|}{ numbers of findings } & \multirow{2}{*}{ estimated FDR } & \multicolumn{2}{|c|}{ true FDR } \\
\hline & & mean & stdev & & mean & stdev \\
\hline \multirow{6}{*}{ 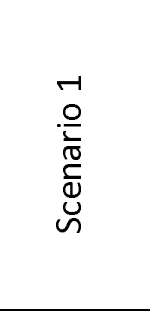 } & eBayesian & 684.3 & 20.74 & 0.049901661 & 0.023415632 & 0.005316 \\
\hline & Exact test & 740.3 & 34.70 & 0.049290389 & 0.086608166 & 0.009598 \\
\hline & GLM & 753.3 & 36.55 & 0.049609439 & 0.096184239 & 0.009912 \\
\hline & DESeq & 552.0 & 24.24 & 0.049794642 & 0.007797678 & 0.002519 \\
\hline & beta t-test & 800.7 & 4.93 & 0.049847667 & 0.144082436 & 0.010284 \\
\hline & mBeta t-test & 726.0 & 3.46 & 0.048788614 & 0.037659939 & 0.008904 \\
\hline \multirow{6}{*}{ 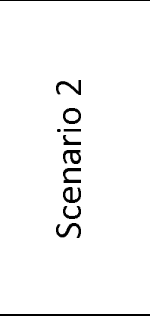 } & eBaysian & 990.7 & 17.61 & 0.04970673 & 0.06424989 & 0.004605 \\
\hline & Exact test & 1003.0 & 28.68 & 0.04971551 & 0.089023333 & 0.002429 \\
\hline & GLM & 1015.3 & 31.34 & 0.049347345 & 0.097436683 & 0.0047 \\
\hline & DESeq & 855.0 & 14.42 & 0.048391895 & 0.011703212 & 0.005338 \\
\hline & beta t-test & 1000.0 & 25.23 & 0.049856733 & 0.155683147 & 0.008473 \\
\hline & mBeta t-test & 957.3 & 12.50 & 0.048912878 & 0.035496106 & 0.007327 \\
\hline \multirow{6}{*}{ 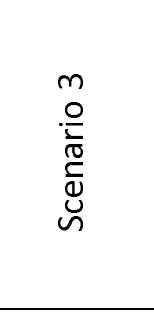 } & eBayesian & 2271.3 & 49.52 & 0.049921947 & 0.027993743 & 0.002909 \\
\hline & Exact test & 2299.3 & 36.69 & 0.049936596 & 0.053499728 & 0.002031 \\
\hline & GLM & 2316.7 & 35.21 & 0.049880522 & 0.058151276 & 0.003686 \\
\hline & DESeq & 1815.7 & 32.02 & 0.049790935 & 0.006585122 & 0.00228 \\
\hline & beta t-test & 2323.0 & 46.11 & 0.049676633 & 0.071477617 & 0.009545 \\
\hline & mBeta t-test & 2093.0 & 32.90 & 0.049291886 & 0.012110596 & 0.002648 \\
\hline \multirow{6}{*}{ 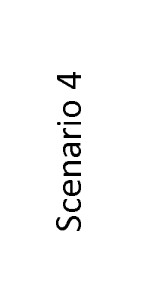 } & eBayesian & 3024.0 & 166.91 & 0.04991893 & 0.070821217 & 0.01752 \\
\hline & Exact test & 2880.3 & 145.08 & 0.049928763 & 0.044367555 & 0.008163 \\
\hline & GLM & 2896.0 & 150.06 & 0.049981628 & 0.048324792 & 0.009311 \\
\hline & DESeq & 2517.7 & 177.80 & 0.049904472 & 0.005894893 & 0.001385 \\
\hline & beta t-test & 2913.0 & 40.92 & 0.049404633 & 0.073357708 & 0.007807 \\
\hline & mBeta t-test & 2789.0 & 41.76 & 0.049425529 & 0.008624077 & 0.002894 \\
\hline
\end{tabular}

Scenario 1: $\mathrm{P}$ (proportion of $\mathrm{DE}$ isoforms) $=10 \%, \mathrm{Q}$ (artificial noise proportion) $=10 \%, \mathrm{~A}$ (condition effect) $=100, R$ (replicate number) $=3$; Scenario 2: $P=10 \%, Q=10 \%, A=300, R=3$; Scenario 3: $P=30 \%, Q=10 \%$, $A=100, R=3$;Scenario 4: $P=30 \%, Q=10 \%, A=300, R=3$. $\omega=1$ for mBeta t-test method in scenarios 1-4. FDR underestimated was marked by red color. 
Table 2 Results of performing statistical methods on simulated data of 18162 isoforms and two conditions in scenarios $5-8$ where simulations were based on real transcriptomic data

\begin{tabular}{|c|c|c|c|c|c|c|}
\hline \multirow{2}{*}{ scenario } & \multirow{2}{*}{$\begin{array}{l}\text { Statistical } \\
\text { methods }\end{array}$} & \multicolumn{2}{|c|}{ number of findings } & \multirow{2}{*}{ estimated FDR } & \multicolumn{2}{|c|}{ true FDR } \\
\hline & & mean & stdev & & mean & stdev \\
\hline \multirow{6}{*}{ 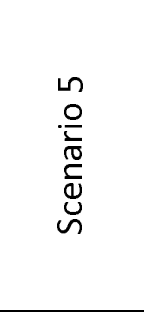 } & eBayesian & 536.0 & 19.31 & 0.049475743 & 0.014225 & 0.004023 \\
\hline & Exact test & 582.7 & 40.15 & 0.049037986 & 0.053917 & 0.022639 \\
\hline & GLM & 592.0 & 41.90 & 0.049785069 & 0.062515 & 0.024089 \\
\hline & DESeq & 400.7 & 8.32 & 0.049205147 & 0.004114 & 0.003743 \\
\hline & Beta t-test & 586.7 & 20.42 & 0.049744667 & 0.165699 & 0.010472 \\
\hline & mBeta t-test & 608.3 & 37.87 & 0.049095016 & 0.045627 & 0.02317 \\
\hline \multirow{6}{*}{ 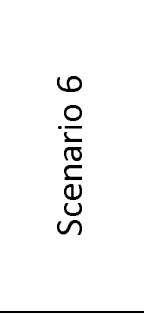 } & eBayesian & 887.3 & 28.91 & 0.049840282 & 0.034131 & 0.002471 \\
\hline & Exact test & 901.0 & 12.76 & 0.049361917 & 0.048886 & 0.005728 \\
\hline & GLM & 911.0 & 13.11 & 0.049262212 & 0.054955 & 0.007382 \\
\hline & DESeq & 746.3 & 19.08 & 0.047905044 & 0.006171 & 0.005225 \\
\hline & Beta t-test & 892.0 & 38.22 & 0.0495794 & 0.13778 & 0.017347 \\
\hline & mBeta t-test & 864.3 & 27.06 & 0.04986485 & 0.027286 & 0.005041 \\
\hline \multirow{6}{*}{ 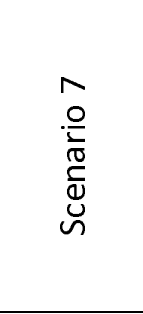 } & eBayesian & 1880.0 & 37.98 & 0.049924036 & 0.016844 & 0.000593 \\
\hline & Exact test & 1905.0 & 63.26 & 0.049760675 & 0.033698 & 0.003521 \\
\hline & GLM & 1936.3 & 67.33 & 0.04987274 & 0.039498 & 0.004252 \\
\hline & DESeq & 1398.0 & 39.39 & 0.049872216 & 0.004724 & 0.002398 \\
\hline & Beta t-test & 1800.7 & 151.30 & 0.0498517 & 0.070921 & 0.011422 \\
\hline & mBeta t-test & 1887.3 & 74.19 & 0.049352367 & 0.018178 & 0.00276 \\
\hline \multirow{6}{*}{ 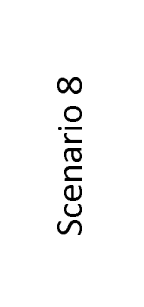 } & ebayesian & 2780.3 & 71.62 & 0.049903333 & 0.049308 & 0.00472 \\
\hline & Exact & 2653.3 & 97.38 & 0.04993212 & 0.033475 & 0.008219 \\
\hline & GLM & 2668.3 & 98.19 & 0.049910444 & 0.036769 & 0.008319 \\
\hline & DESeq & 2275.3 & 81.32 & 0.049506184 & 0.004339 & 0.002514 \\
\hline & Beta t-test & 2509.7 & 40.82 & 0.0498865 & 0.060843 & 0.010993 \\
\hline & mBeta t test & 2599.7 & 29.73 & 0.049559249 & 0.014071 & 0.004408 \\
\hline
\end{tabular}

781 Scenario 5: $P=10 \%, Q=30 \%, A=100, R=3$; Scenario 6: $P=10 \%, Q=30 \%, A=300, R=3$; Scenario 7: $P=30 \%$,

$782 \mathrm{Q}=30 \%, \mathrm{~A}=100, \mathrm{R}=3$; Scenario 8: $\mathrm{P}=30 \%, \mathrm{Q}=30 \%, \mathrm{~A}=300, \mathrm{R}=3 . \omega=1$ for mBeta t-test method in scenarios 783 5-8. FDR underestimated was marked by red color. 
Table 3 Results of performing statistical methods on simulated data of 18162 isoforms and two conditions in scenarios 9-12 where simulations were based on real isoform transcriptomic data

\begin{tabular}{|c|c|c|c|c|c|c|}
\hline \multirow[b]{2}{*}{ scenario } & \multirow{2}{*}{$\begin{array}{c}\text { Statistical } \\
\text { method }\end{array}$} & \multicolumn{2}{|c|}{ number of findings } & \multirow[b]{2}{*}{ estimated FDR } & \multicolumn{2}{|c|}{ true FDR } \\
\hline & & mean & stdev & & mean & stdev \\
\hline \multirow{6}{*}{ 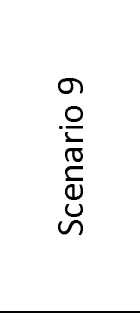 } & eBayesian & 1466.7 & 136.99 & 0.049816998 & 0.065909 & 0.017935 \\
\hline & Exact test & 1455.7 & 135.50 & 0.04942623 & 0.075021 & 0.023157 \\
\hline & GLM & 1492.0 & 157.43 & 0.049120039 & 0.092843 & 0.032046 \\
\hline & DESeq & 1270.7 & 116.81 & 0.04968407 & 0.016749 & 0.004976 \\
\hline & Beta t-test & 1447.7 & 103.58 & 0.049672 & 0.116815 & 0.028366 \\
\hline & mBeta t-test & 1457.3 & 114.02 & 0.048886388 & 0.03596 & 0.009967 \\
\hline \multirow{6}{*}{ 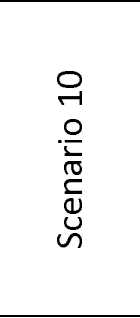 } & eBayesian & 1822.3 & 95.61 & 0.049864672 & 0.104564 & 0.013078 \\
\hline & Exact test & 1768.3 & 117.50 & 0.049594284 & 0.08398 & 0.028075 \\
\hline & GLM & 1794.7 & 131.76 & 0.049606781 & 0.094059 & 0.033016 \\
\hline & DESeq & 1593.3 & 125.52 & 0.049260009 & 0.021667 & 0.012233 \\
\hline & Beta t-test & 1772.0 & 67.55 & 0.049848 & 0.132159 & 0.025635 \\
\hline & mBeta t-test & 1697.667 & 67.26 & 0.049301841 & 0.032401 & 0.007489 \\
\hline \multirow{6}{*}{ 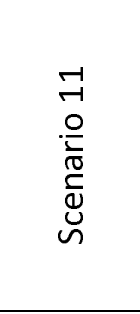 } & eBayesian & 4894.7 & 289.89 & 0.049930905 & 0.081428 & 0.011789 \\
\hline & Exact test & 4633.3 & 303.32 & 0.049761768 & 0.051046 & 0.013995 \\
\hline & GLM & 4681.3 & 332.24 & 0.049960589 & 0.057404 & 0.018121 \\
\hline & DESeq & 4206.3 & 317.16 & 0.049814792 & 0.013886 & 0.005984 \\
\hline & Beta t-test & 4700.7 & 362.29 & 0.0493765 & 0.070815 & 0.014574 \\
\hline & mBeta t-test & 4438.3 & 149.79 & 0.04952921 & 0.0124 & 0.000406 \\
\hline \multirow{6}{*}{ 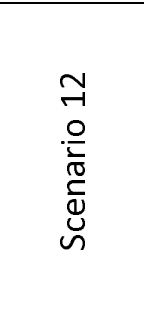 } & ebayesian & 5701.0 & 206.72 & 0.049939398 & 0.099753 & 0.01504 \\
\hline & Exact test & 5453.7 & 100.48 & 0.049737062 & 0.048492 & 0.006854 \\
\hline & GLM & 5454.7 & 145.40 & 0.049840291 & 0.053679 & 0.008784 \\
\hline & DESeq & 4915.3 & 228.00 & 0.049430541 & 0.012464 & 0.005037 \\
\hline & Beta t-test & 5091.7 & 128.48 & 0.049957167 & 0.066915 & 0.013236 \\
\hline & mBeta t-test & 5072.3 & 135.57 & 0.049633984 & 0.01087 & 0.002098 \\
\hline
\end{tabular}

792

793

794

795

796

797

798

799

800

801
Scenario 9: $P=10 \%, Q=10 \%, A=100, R=5$; Scenario 10: $P=10 \%, Q=10 \%, A=300, R=5$; Scenario 11: $P=30 \%$, $Q=10 \%, A=100, R=5$; Scenario $4: P=30 \%, Q=10 \%, A=300, R=5 . \omega=0.45$ for the mBeta t-test method in scenarios 9-12. FDR underestimated was marked by red color. 
Table 4 Stability analysis of methods

\begin{tabular}{|c|c|c|c|}
\hline \multicolumn{2}{|c|}{ Standard deviation of findings } & \multicolumn{2}{|c|}{ Standard deviation of true FDR } \\
\hline method & Averaged order score & method & Averaged order score \\
\hline mBeta t-test & 2.333333 & DESeq & 1.833333 \\
\hline Exact test & 3.166667 & mBeta t-test & 2.5 \\
\hline Beta t-test & 3.25 & eBayesian & 3 \\
\hline eBayesian & 3.416667 & Exact test & 3.5 \\
\hline DESeq & 3.833333 & Beta t-test & 5.083333 \\
\hline GLM & 5 & GLM & 5.083333 \\
\hline
\end{tabular}

804

805

806

807

808

809

810

811

812

813

814

815

816

817

818

819

820

821 
Table 5 Averaged work efficiencies of statistical methods

\begin{tabular}{|c|c|c|c|c|c|c|c|c|c|c|c|c|}
\hline \multirow[b]{2}{*}{ Scenario factors } & \multicolumn{2}{|c|}{ eBayesian } & \multicolumn{2}{|c|}{ Exact test } & \multicolumn{2}{|c|}{ GLM } & \multicolumn{2}{|c|}{ DESeq } & \multicolumn{2}{|c|}{ Beta t-test } & \multicolumn{2}{|c|}{ mBeta t-test } \\
\hline & mean & stdev & mean & stdev & mean & stdev & mean & stdev & mean & stdev & mean & stdev \\
\hline 3 replicate libraries & 0.48697 & 0.321 & 0.3726 & 0.40689 & 0.27557 & 0.3884 & 0.57566 & 0.15142 & 0 & 0 & 0.69207 & 0.12025 \\
\hline $\begin{array}{l}5 \text { replicate libraries } \\
\text { proportion of } D E\end{array}$ & 0 & 0 & 0.46259 & 0.53764 & 0 & 0 & 0.81272 & 0.15142 & 0 & 0 & 0.87067 & 0.07198 \\
\hline $\begin{array}{l}\text { genes/isoforms }=10 \% \\
\text { proportion of } D E\end{array}$ & 0.30974 & 0.35327 & 0.13241 & 0.32434 & 0 & 0 & 0.63812 & 0.09414 & 0 & 0 & 0.75333 & 0.14388 \\
\hline $\begin{array}{l}\text { genes } / \text { isoforms }=30 \% \\
\text { artificial effect }\end{array}$ & 0.3281 & 0.36791 & 0.49808 & 0.42951 & 0.16917 & 0.31848 & 0.66774 & 0.18908 & 0 & 0 & 0.7625 & 0.14959 \\
\hline $\begin{array}{l}\text { proportion }=10 \% \\
\text { artificial effect }\end{array}$ & 0.31775 & 0.36784 & 0.21165 & 0.42329 & 0.2128 & 0.42559 & 0.62857 & 0.19842 & 0 & 0 & 0.7298 & 0.1188 \\
\hline $\begin{array}{l}\text { proportion }=30 \% \\
\text { condition effect }\end{array}$ & 0.3281 & 0.36791 & 0.49808 & 0.42951 & 0.16917 & 0.31848 & 0.66774 & 0.13814 & 0 & 0 & 0.7625 & 0.14959 \\
\hline $\begin{array}{l}=100 \\
\text { condition effect }\end{array}$ & 0.3827 & 0.30323 & 0.23505 & 0.37554 & 0.09485 & 0.23234 & 0.5427 & 0.19842 & 0 & 0 & 0.66057 & 0.12078 \\
\hline$=300$ & 0.2666 & 0.41316 & 0.57015 & 0.44847 & 0.27258 & 0.4228 & 0.76666 & 0.1635 & 0 & 0 & 0.84263 & 0.07676 \\
\hline
\end{tabular}


Table 6. Results of performances of 3 statistical methods on two real gene transcriptomic data

\begin{tabular}{c|c|c|c|c}
\hline data type & & Exact test & mBeta t-test & Beta t-test \\
\hline \multirow{4}{*}{ Gene count data } & \# of DE genes found & 799 & 1774 & 2515 \\
\cline { 2 - 5 } & estimated FDR & 0.0499 & 0.0499 & 0.0489 \\
\cline { 2 - 5 } & least true FDR & 0.2753 & 0.0056 & 0.3062 \\
\hline \multirow{4}{*}{ Isoform count data } & \# of DE isoforms found & 1029 & 1981 & 3025 \\
\cline { 2 - 5 } & estimated FDR & 0.0499 & 0.0498 & 0.0499 \\
\cline { 2 - 5 } & least true FDR & 0.2799 & 0.0101 & 0.3530 \\
\hline
\end{tabular}

a: $\omega=1$ 